\title{
Nitrogen-vacancy centers promote super-radiant maser performance
}

\author{
Chen Qiu ${ }^{1}$ and Hui-Xiong Deng ${ }^{2 *}$
}

The technological application of masers, i.e., the so-called microwave amplification by stimulated emission of radiation (Fig. 1), is restricted by insatiable operating conditions, including deep-freezing and high-vacuum environments. The masers that can be operated at room temperature are enticing but difficult to achieve [1-6]. This is because the lifetime of emitters (electron spins) in solids is ordinarily transitory, that is, the spin population inversion is exceedingly fast $(\sim \mathrm{ns})$ [7,8]. Interestingly, the nitrogen-vacancy $\left(\mathrm{NV}^{-1}\right)$ center, which has been widely studied in quantum computing, stands out for its robustness. Its quantum state (spin up or spin down) can be initialized and manipulated, featuring the longest known solid state spin lifetime $(\sim 5 \mathrm{~ms})$ at room temperature [9]. Indeed, the room-temperature maser based on the $\mathrm{NV}^{-1}$ center in diamond has been reported experimentally, demonstrating the Rabioscillations, super-radiant pulses and stimulated emission $[2,4,5]$. These phenomena indicate the nature of collective and strong coupling in the optical frequency range, which is a prerequisite for the observation of super-radiant lasing. Therefore,

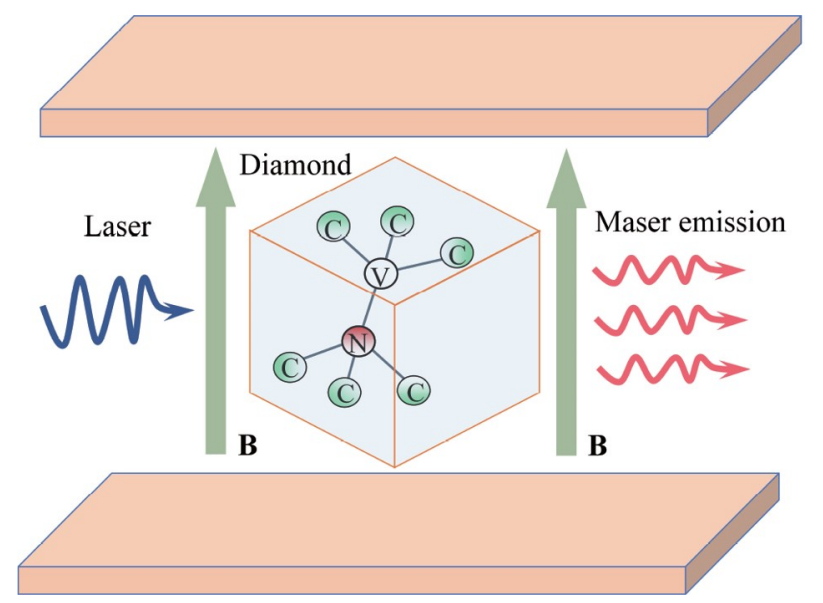

Figure 1 Schematic of a room-temperature maser with nitrogen-vacancy $\left(\mathrm{NV}^{-1}\right)$ center spins. This picture illustrates a diamond being irradiated by an optically excited laser and subjected to a static magnetic field $\mathbf{B}$ in a microwave resonator. The insert figure shows the configuration of the $\mathrm{NV}^{-1}$ center, where green and red balls represent carbon and nitrogen atoms, respectively, and the white ball is a vacancy. it is crucial to investigate how the collective and strong coupling $\mathrm{NV}^{-1}$ spin achieves a super-radiant maser [1].

In a paper entitled "A superradiant maser with nitrogenvacancy center spins" [1], the authors take advantage of the numerical solution of the second-order mean-field equation and demonstrate that if the spin-ensemble is pumped beyond a threshold rate, then the linewidth of a super-radiant maser can be lowered to sub-millihertz, and remain below kilohertz at room temperature for sufficient spins. Most importantly, they conceive the dominant role of the coherence and super-radiant maser in $\mathrm{NV}^{-1}$ spin-ensemble, which may challenge the Schawlow-Townes limit [3].

As room-temperature masers may find wide applications in deep-space communications and radio astronomy and play a key role in realizing quantum programming in quantum computers composed of superconducting circuits, a deep understanding of $\mathrm{NV}^{-1}$ spin-ensemble becomes especially critical. This work has made an outstanding contribution to the development of roomtemperature masers and will help us further understand superradiant masers.

Received 10 November 2021; accepted 19 November 2021; published online 3 December 2021

1 Wu Q, Zhang Y, Yang X, et al. A superradiant maser with nitrogenvacancy center spins. Sci China-Phys Mech Astron, 2021, 65: 217311

2 Breeze JD, Salvadori E, Sathian J, et al. Continuous-wave room-temperature diamond maser. Nature, 2018, 555: 493-496

3 Schawlow AL, Townes CH. Infrared and optical masers. Phys Rev, 1958, 112: 1940-1949

4 Liu C, Mucci M, Cao X, et al. Proposal for a continuous wave laser with linewidth well below the standard quantum limit. Nat Commun, 2021, 12: 5620

5 Jin L, Pfender M, Aslam N, et al. Proposal for a room-temperature diamond maser. Nat Commun, 2015, 6: 8251

6 Breeze J, Tan KJ, Richards B, et al. Enhanced magnetic Purcell effect in room-temperature masers. Nat Commun, 2015, 6: 6215

$7 \mathrm{Yu}$ FS. Role of decay in the search for double-charm baryons. Sci China-Phys Mech Astron, 2020, 63: 221065

8 Mallamace F, Corsaro C, Mallamace D, et al. The Boson peak interpretation and evolution in confined amorphous water. Sci China-Phys Mech Astron, 2019, 62: 107004

9 Weber JR, Koehl WF, Varley JB, et al. Quantum computing with defects. Proc Natl Acad Sci USA, 2010, 107: 8513-8518

\footnotetext{
${ }^{1}$ Beijing Computational Science Research Center, Beijing 100094, China

${ }^{2}$ State Key Laboratory of Superlattices and Microstructures, Institute of Semiconductors, Chinese Academy of Sciences, Beijing 100083, China

* Corresponding author (email: hxdeng@semi.ac.cn)
} 